\title{
MMSE-V-BLAST Optimal-Ordering In Different Channels
}

\author{
Amit Grover ${ }^{1 *}$, Neeti Grover ${ }^{2}$ \\ $I^{*}$ (Department of Electronics and Communication Engineering, Shaheed Bhagat Singh State Technical \\ Campus, Moga Road (NH-95), Ferozepur-152004, India. \\ ${ }^{2}$ (Department of Applied Sciences and Humanities, Shaheed Bhagat Singh State Technical Campus, Moga \\ Road (NH-95), Ferozepur-152004, India)
}

\begin{abstract}
The Bell Labs layered space-time (BLAST) algorithm is simple, and hence, a popular choice for a multiple-input-multiple-output (MIMO) receiver. Because of the difficulties that arise due to the optimal ordering in terms of BER, while considering real analytical evaluations, the one and only solution is by introducing OSIC schemes to improve the performance of the system in terms of BER. In this article we are improving the performance of the system in terms of BER; by introducing OSIC schemes along with the Minimum Mean Square Error detector that also combat the error propagation of the system. We have also analyzed the BER performance of these MIMO schemes using different modulation techniques like BPSK, QPSK and 16 QAM with different antenna configurations in classical independent identically distributed (i.i.d) Rayleigh fading channel and Rician fading channel. Finally we observed that BPSK and QPSK modulation techniques give the almost same result in VBLAST with the given detection technique in both the channels and 16-QAM modulation technique gives the worst result. We have also concluded that as we keeping number of receiving antennas more than transmitting antenna we get better BER performance that means we can remove the more errors. If number of transmitting antennas are more than receiving antennas we get worst BER performance that means we can remove fewer errors.
\end{abstract}

Keywords:- Binary Phase Shift Key (BPSK), Bit Error Rate (BER), Multiple input multiple output (MIMO), Minimum Mean Square Error (MMSE), Ordered Successive Interference Cancellation (OSIC), Quardrature Phase Shift Keying (QPSK),Quadrature Amplitude Modulation (QAM), Independent identically distributed (i.i.d), Bell Labs layered space-time (BLAST).

\section{Introduction}

The use of multiple antennas at both the transmitter and the receiver sides can drastically improve the channel capacity and data rate [8]. The study of the performance limits of MIMO system [3] becomes very important since it will give lot ideas in understanding and designing the practical MIMO systems [4].Many schemes have been proposed to explode the high spectral efficiency of MIMO channels, among which VBLAST [6] is relatively simple and easy to implement and can achieve a large spectral efficiency. In V-BLAST [5] the parallel transmission of the different data streams has been carried out by placing each input data stream into different independent sub streams over the ' $n$ ' transmitting antennas. At the receiver end, antennas receive the sub-streams, which are mixed and superimposed by noise. Detection process [5] mainly involves three operations: Interference Suppression (nulling), interference cancellation (Subtraction) and Optimal Ordering. The interference nulling process is carried out by projecting the received signal into the null subspace spanned by the interfering signals. The interference cancellation process is done by subtracting the detected symbols from the received vectors. The optimal Ordering is the last process that ensures the detected symbol has highest Signal to noise ratio (SNR). So, V-BLAST algorithm [6] integrates both linear and non-linear algorithms presented in the interference nulling and interference cancellation respectively. ${ }^{~} \eta$ an independent, identically distributed (i.i.d) Flat fading Ricean channel [1] with ' $N$ ' transmitting antennas and ' $M$ ' receiving antennas In this we will considered receiving antennas are greater than or equal to transmitting antennas $(M \geq N)$, the first detected sub-stream has a diversity gain of only M-N+1 [2].

\section{MIMO Channel Model}

Let us consider a communication system with ' $N$ ' number of transmitting antennas and ' $M$ ' number of receiving antennas in an i.i.d Ricean Flat Fading channel [1]. The sampled baseband representation of signal is given

by

$$
\mathrm{y}=\mathrm{Hx}+\mathrm{n}
$$


And the complex baseband representation of signal [7] is given by

$$
y=\sqrt{\frac{P}{M}} H x+n
$$

where $y \in C^{N \times 1}$ is the received signal vector, $x \in C^{M \times 1}$ is the transmitted signal vector with zero mean and unit variance, $\mathrm{P}$ is the total transmit power, $H \in C^{N \times M}$ is the channel response matrix with possibly correlated fading coefficients. In order to access the performance of V-BLAST in correlated channel, we adopted a correlation-based channel model which is expressed as

$$
H \sim R_{R x}^{\frac{1}{2}} H_{w}\left(R_{T x}^{1 \backslash 2}\right)^{T}
$$

where $\mathrm{x} \sim \mathrm{y}$ denotes that $\mathrm{x}$ and $\mathrm{y}$ are identical in distribution, $R_{R x}$ and $T_{T x}$ are the normal correlation distribution matrices at the $\mathrm{R}_{\mathrm{x}}$ and transmitter $\left(\mathrm{T}_{\mathrm{x}}\right)$ respectively, and $H_{W} \in C^{N X M}$ contains i.i.d complex Gaussian entries with zero mean and unit variance.

\section{Fading Channel}

Fading is used to describe the rapid fluctuations of the amplitudes, phases or multipath delays of a radio signal over a short period of time or travel distance, so that large scale path loss effect may be ignored

\subsection{Rayleigh Fading Channel}

The fading effect is usually described statistically using the Rayleigh distribution [10].

\subsection{Rician Fading Channel}

The presence of a fixed (possibly line-of-sight or LOS) component in the channel will result in Ricean fading [1].In the presence of an LOS component between the transmitter and the receiver, the MIMO channel may be modeled as the sum of a fixed component and a fading component and given by following equation

$$
H=\sqrt{\frac{k}{1+k}} \bar{H}+\sqrt{\frac{k}{1+k}} H_{w}
$$

$\sqrt{\frac{\mathrm{k}}{1+\mathrm{k}}} \overline{\mathrm{H}}=\mathrm{E}[\mathrm{H}]$ is the LOS component of the channel.

$\sqrt{\frac{\mathrm{k}}{1+\mathrm{k}}} \mathrm{H}_{\mathrm{w}}$ is the fading component.

$\mathrm{k} \geq 0$ in equation is the Ricean $\mathrm{k}$-factor and when $\mathrm{k}=0$, we have pure Rayleigh fading channel and $\mathrm{k}=\infty$ corresponds to a non-fading channel.

\section{Decoding Algorithm for V-BLAST System}

In decoding algorithm, the strongest symbol is detected in the first step and second step is to cancel the effects of this strongest symbol from all received signals and in third step algorithm detects the next strongest symbol. This process has been repeated until all the symbols are detected with the optimal detection order from the strongest symbol to the weakest one. This is the original decoding algorithm [2] of V-BLAST preset. It only works if the number of receive antennas is more than the number of transmit antennas, that is $\mathrm{M} \mathrm{x} \mathrm{N}$. Decoding Algorithm of V-BLAST is shown in Figure.1

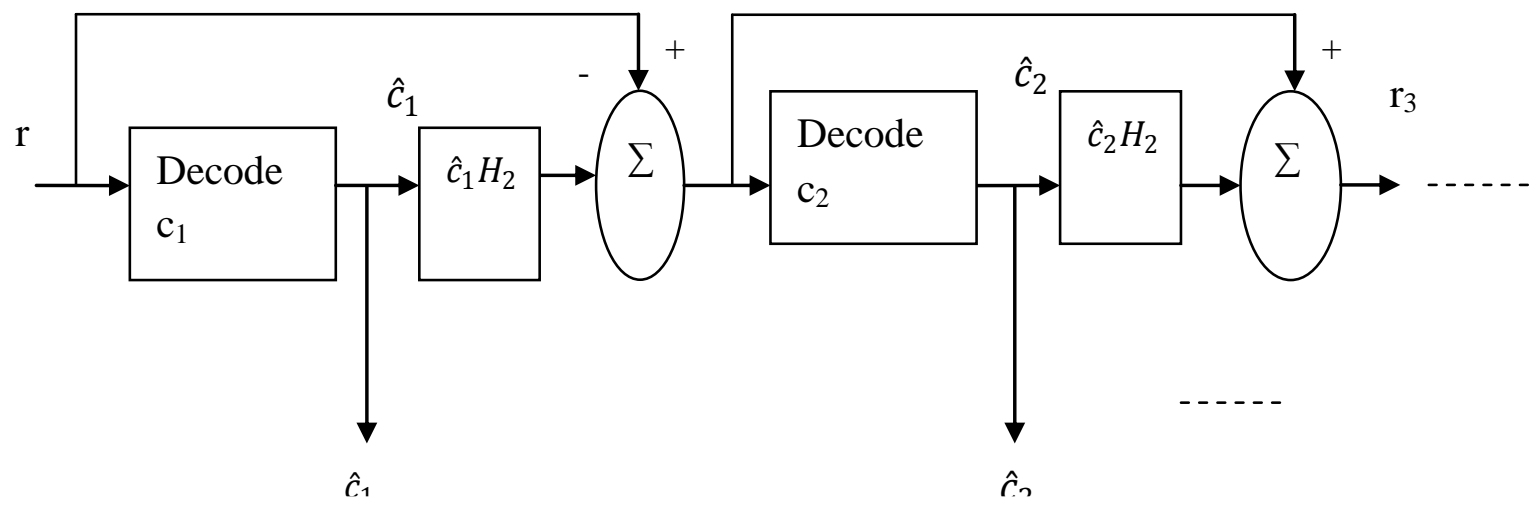

Fig.1 VBLAST Decoder block diagram 
The algorithm includes three steps:

- ordering;

- interference cancellation;

- Interference nulling.

\subsection{Ordering}

In decoding the first symbol, the interference from all other symbols is considered as noise. After finding the best candidate for the first symbol, the effects of this symbol in all of the receiver equations are canceled. The process continues until all symbols are detected. Of course, the order in which the symbols are detected will impact the final solution.

\subsection{Interference Cancellation}

At stage $\mathrm{n}$ of the algorithm, when $c_{n}$ is being detected, symbols $c_{1}, c_{2}, \ldots, c_{n-1}$ have been already detected. Let us assume a perfect decoder, that is the decoded symbols $\widehat{c_{1}}, \widehat{c_{2}}, \ldots \ldots, \hat{c}_{n-1}$ are the same as the transmitted symbols $c_{1}, c_{2}, \ldots, c_{n-1}$.

Therefore, at the $n^{\text {th }}$ stage of the algorithm after detecting the nth symbol as $\hat{c}_{n}[10]$, its effect is canceled from the equations by

$$
r_{n+1}=r_{n}+\hat{c}_{n} H_{n}
$$

\subsection{Interference Nulling}

In this step the nth symbol is detected by nulling the interference caused by symbols $\boldsymbol{c}_{\boldsymbol{n}+1}, \boldsymbol{c}_{\boldsymbol{n}+2}, \ldots, \boldsymbol{c}_{\boldsymbol{N}}$. Like any other interference suppression problem, there are many different methods to detect a symbol in the presence of interference.

\section{MMSE-V-BLAST Decoder}

The MMSE receiver suppresses both the interference and noise components. At low SNR, MMSE becomes matched filter. For MMSE-V-BLAST, the nulling vector for the $\mathrm{i}^{\text {th }}$ layer is

$$
w^{i}=\left(H_{i} H_{i}^{*}+\frac{1}{s n r} I\right)^{-1} h_{i}, \quad \mathrm{i}=1,2 \ldots \ldots \mathrm{N}
$$

Where $H_{i}=C^{M \times i}$ consists of the first I columns of $\mathrm{H}$.

\subsection{Minimum Mean Square Error (MMSE) With SIC}

In order to consider OSIC with MMSE, Covariance matrix of the estimation error $\left(s-s_{e s t}\right) \square \square$ will be used to determine good ordering for detection and can be written as

$Q=E\left[\left(s-s_{\text {est }}\right)\left(s-s_{\text {est }}\right)^{H}\right]=\sigma_{n}^{2}\left(\alpha I+H^{H} H\right)^{-1} \equiv \sigma_{n}^{2} P$

And the procedure considered for this will be explained in these three steps:

1) Compute $\mathrm{W}$ ( $\mathrm{P}$ is obtained while determining $\mathrm{W}$ ). Find the smallest diagonal entry of $\mathrm{P}$ and suppose this is the $\mathrm{p}$-th entry. Permute the $\mathrm{p}$-th column of $\mathrm{H}$ to be last column and permute the rows of $\mathrm{W}$ accordingly.

2) From the estimate of the corresponding elements of $s$.

In case of MMSE: $($ ses $)=W M x$

Where the weight vector equals row $\mathrm{M}$ (number of transmitting antennas) of the permuted $\mathrm{W}$

3) While M-1>0 go back to step 1, but now with: $H \rightarrow H M-1=h 1 \ldots \ldots \ldots h M-1$ So here we can see that we get optimal ordering by using MMSE with OSIC A bank of linear MMSE receivers, each estimating one of the parallel data streams, with streams successively cancelled from the received vector at each stage can be easily explained with block diagram in figure. 2 


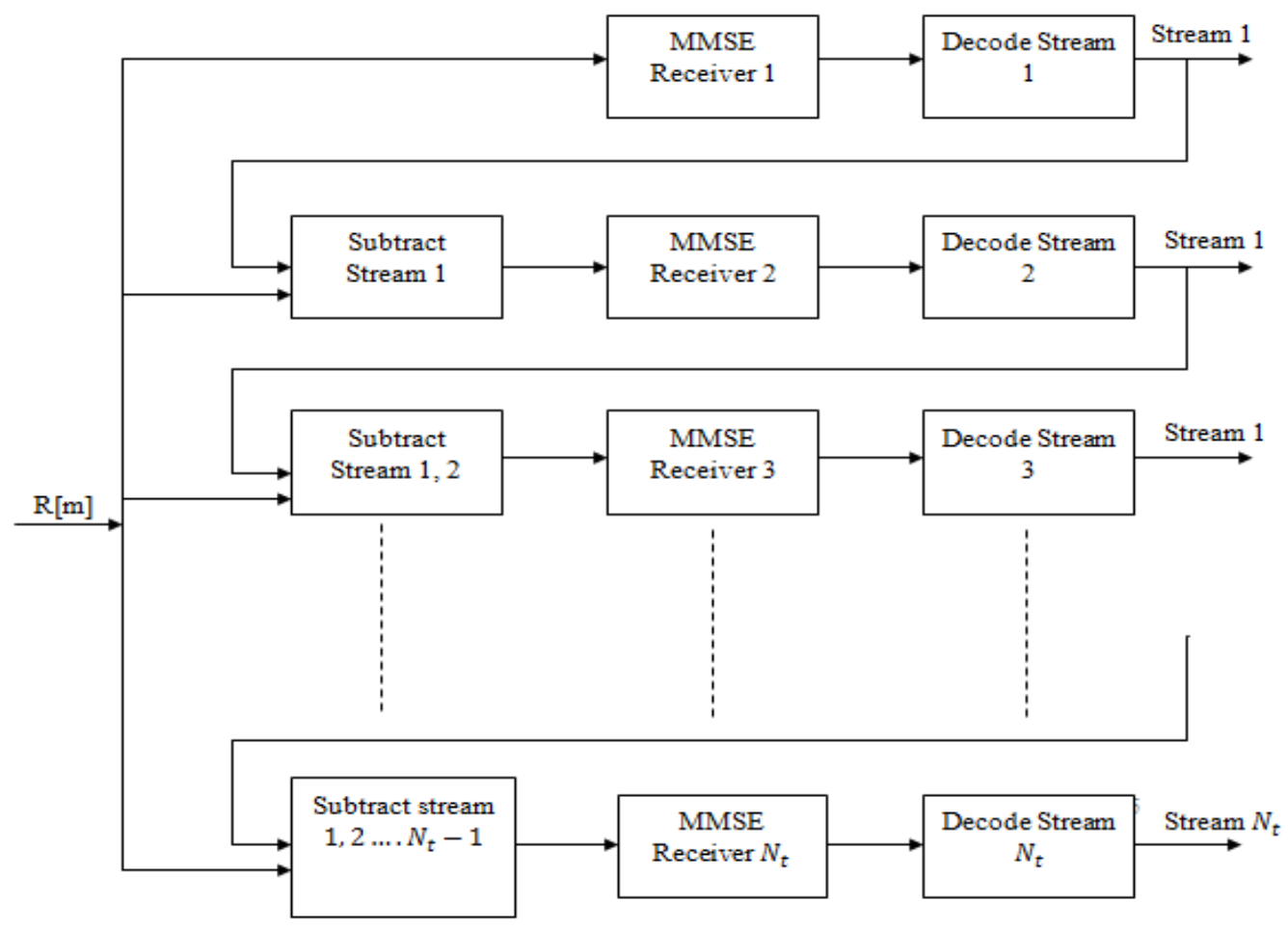

Fig.2 SIC MMSE detector

\section{Simulation and Results}

We have done the simulation using MATLAB. Different graphs show the performance of MMSE detector with and without ordering schemes, which proves the better performance of the given detector using optimal ordering. By considering the different antenna configurations with the modulation techniques like BPSK and QPSK, the performance of the MMSE detector has been considered, which shows by keeping more number of antennas at the receiver the performance has been improved as compare to the number of antennas at the transmitter side.
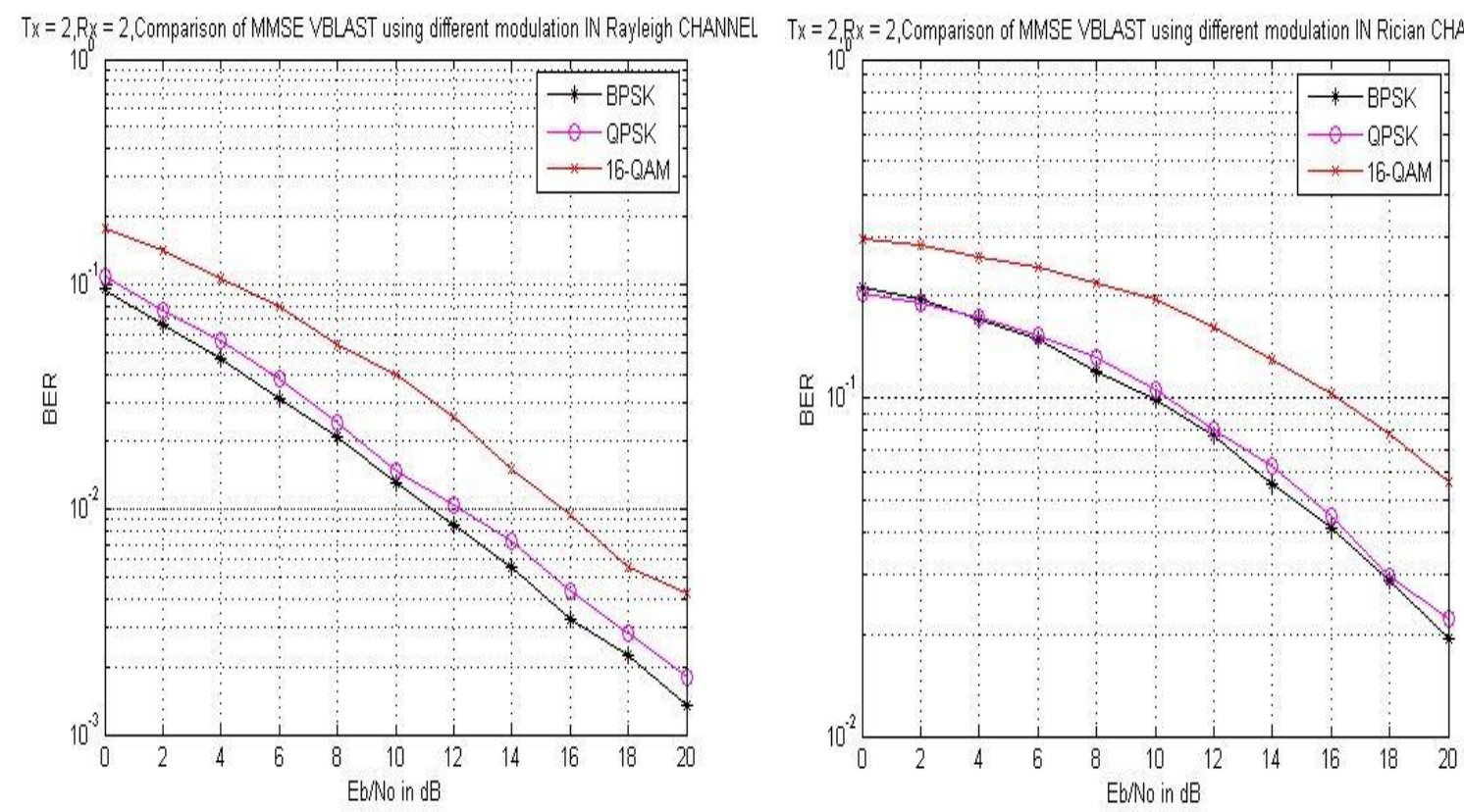

Fig.3 and Fig.4 Shows Comparison of different modulations in MMSE V-BLAST in Rayleigh and Rician Channel 
In Figure3, we have observed that BPSK and QPSK have almost the same results and 16 QAM has the worst result than BPSK and QPSK. At BER 0.001, there is approximately $5 \mathrm{~dB}$ difference between the BPSK and16 QAM modulations in MMSE in Rayleigh Channel.

In Figure4, we have observed that BPSK and QPSK have almost the same results and 16 QAM has the worst result than BPSK and QPSK. At BER 0.01, there is approximately $6 \mathrm{~dB}$ difference between the BPSK and16 QAM modulations in MMSE in Ricean Channel.

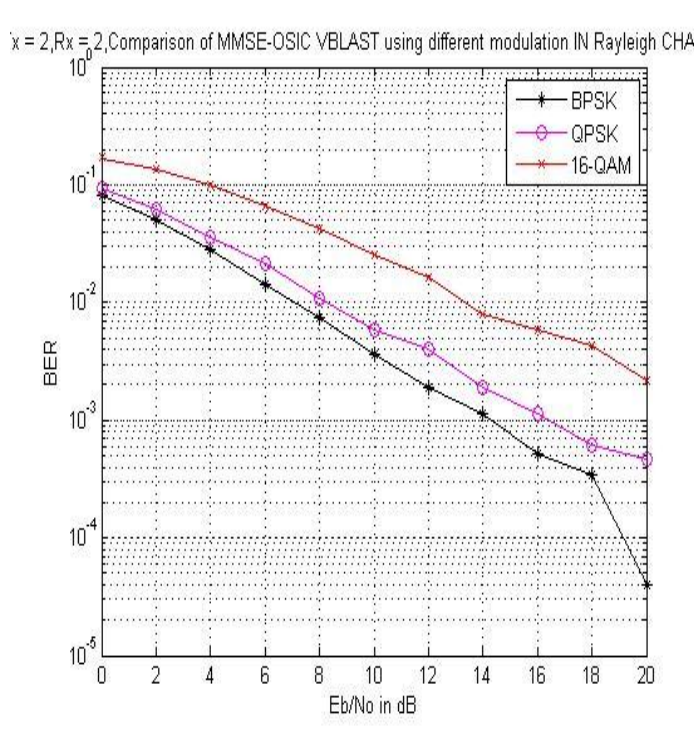

Fig. 5

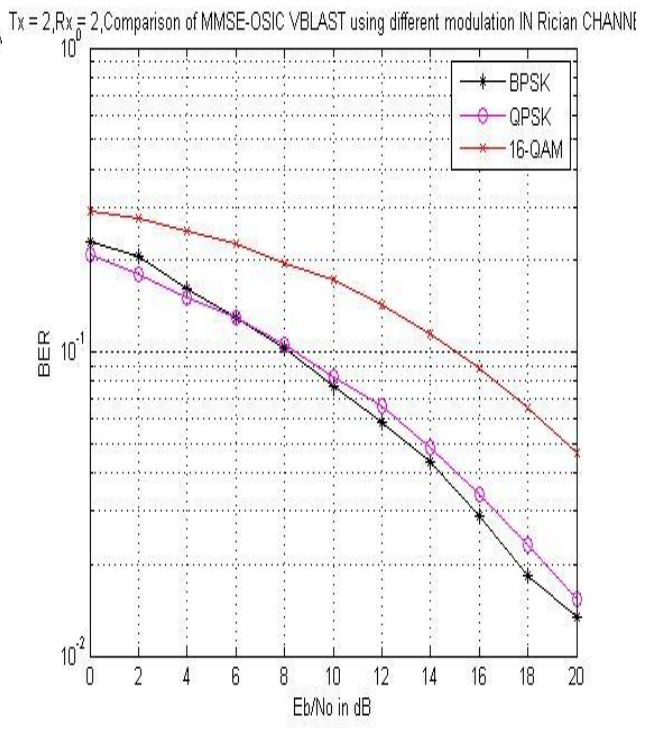

Fig.6

In Figure5, we have observed that BPSK and QPSK have almost the same results and 16 QAM has the worst result than BPSK and QPSK. At BER 0.001, there is approximately $7 \mathrm{~dB}$ difference between the BPSK and 16 QAM modulations in MMSE-OSIC in Rayleigh Channel. In Figure6, we have observed that BPSK and QPSK have almost the same results and 16 QAM has the worst result than BPSK and QPSK. At BER 0.01, there is approximately $8 \mathrm{~dB}$ difference between the BPSK and16 QAM modulations in MMSE-OSIC in Ricean Channel.

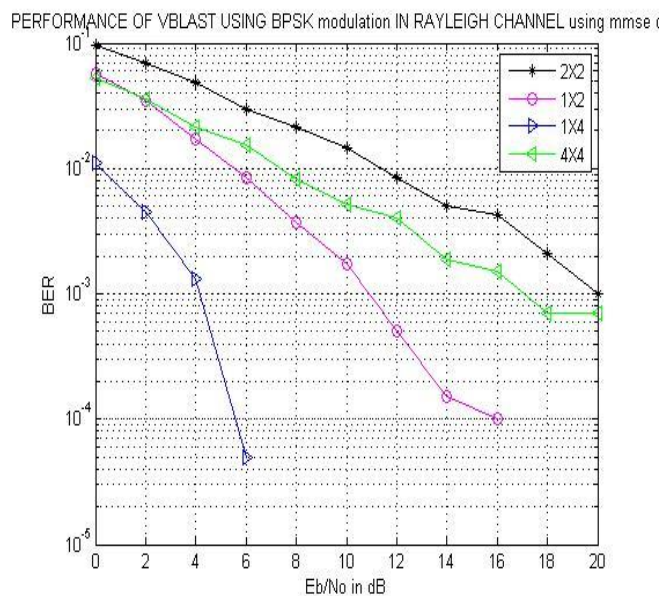

Figure.7: BER for MMSE-VBLAST using BPSK modulation in Rayleigh Channel

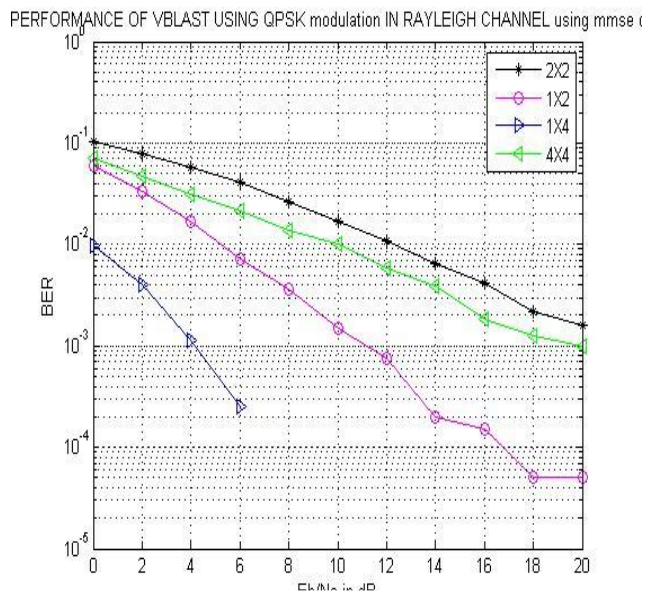

Figure.8: BER for MMSE-VBLAST using QPSK modulation in Rayleigh Channel 


\begin{tabular}{|l|l|}
\hline $\mathbf{M} \mathbf{X} \mathbf{N}$ & BER \\
\hline $1 \mathrm{X} 4$ & 0.000398 \\
\hline $4 \mathrm{X} 4$ & 0.0050 \\
\hline $1 \mathrm{X} 2$ & 0.050 \\
\hline $2 \mathrm{X} 2$ & 0.01 \\
\hline
\end{tabular}

Table.1: BER for MMSE-VBLAST using BPSK modulation in Rayleigh Channel at $\mathrm{SNR}=2 \mathrm{~dB}$

\begin{tabular}{|l|l|}
\hline M X N & BER \\
\hline $1 \mathrm{X} 4$ & .000398 \\
\hline $4 \mathrm{X} 4$ & 0.050 \\
\hline $1 \mathrm{X} 2$ & 0.025 \\
\hline $2 \mathrm{X} 2$ & 0.079 \\
\hline
\end{tabular}

Table.2: BER for MMSE-VBLAST using QPSK modulation in Rayleigh Channel at $\mathrm{SNR}=2 \mathrm{~dB}$

From Figure.7 we conclude that $1 \times 4$ antenna configuration gets an optimal result than another antenna configurations using BPSK modulation in Rayleigh channel and the table.1demonstrates this by showing the values of the BER of the different antenna configurations, in which $1 \times 4$ antenna configuration has minimum BER. So we conclude that $1 \times 4$ configuration gives the best result for MMSE-VBLAST decoder in the Rayleigh Channel.

From Figure. 8 we conclude that $1 \times 4$ antenna configuration gets an optimal result than another antenna configurations using QPSK modulation in Rayleigh channel and the table.2 demonstrates this by showing the values of the BER of the different antenna configurations, in which at SNR of 2dB, 1X4 antenna configuration has the minimum value of 0.00398 as BER than other antenna configurations. So we conclude that $1 \times 4$ configuration gives the best result for MMSE-VBLAST decoder in the Rayleigh Channel.

\section{Conclusions}

By introducing the OSIC schemes the performance of VBLAST architecture with the MMSE detector has been improved. OSIC schemes also improve the V-BLAST system by combating the error propagation. Furthermore we observed that BPSK and QPSK modulation techniques give the almost same results in VBLAST with the given detection technique in both the channels and 16-QAM modulation technique gives the worst results. Finally we concluded that as we keeping number of receiving antennas more than transmitting antenna we get better BER performance. If number of transmitting antennas is more than receiving antennas we get worst BER performance.

\section{References}

[1] R.U. Nabar, H.Boleskei and A.J. Paulraj (2005)," Diversity and outage performance in Space Time Block Coded Rician MIMO Channels” IEEE Trans. Wireless Commun. Vol.4, pp.1102-1111.

[2] G.Ginis and J.M.Cioffi (2001), "On the relationship between V-BLAST and GDFE," IEEE Communications letters, vol. 5, pp. 364-366.

[3] R. U. Nabar A. J. Paulraj, D. A. Gore and H. Bolcskei (2004), "An overview of MIMO communications-a key to gigabit wireless," Proceedings of the IEEE, vol. 92, no. 2, pp. 198-218.

[4] A.Paulraj and R.J.Heath (2001), "Characterization of MIMO Channels for Spatial Multiplexing Systems "IEEE International Conference on Communications, vol.2, no.11-14, pp-591-595.

[5] G.D.Golden, G.J.Foschini, R.A. Valenzuela, anu P.W.Wolniasky (1999), "Detection algorithm and initial laboratory results using the V-BLAST space-time communication architecture," Electron Lett., vol.35, no. 1, pp.1415.

[6] P.Wolniosky, G.J.Foschini, G.D.Golden and R.A. Valenzuela (1998)," V-BLAST: An Architecture for realizing very high data rates over rich scattering wireless channel" URSI International Symposium on Signals, Systems and Electronics, ISSSE 98.

[7] Choi, J, Yu,H., and Lee, Y.H.(2005), "Adaptive MIMO decision Feedback Equalization for Receivers with time varying channels", IEEE transaction on signal processing, vol.55, No. 7, pp.3405-341_

[8] I.E. Telatar (1999), "Capacity of multi-antenna Gaussian channels, "European Transactions on Telecommunications, vol. 10, no.6, pp.585-595.

[9] M.Varanasi and T.Guess (1997), "Optimum decision feedback multiuser equalization with successive decoding achieves the total capacity of the Gaussian multiple-access channel," Conference Record of the Thirty-First Asilomar Conference on signals, Systems and computers, vol. 2, pp. 1405-1409.

[10] Sukhchain Singh, Gurpreet Singh and Amit Grover (2012), "Performance Evaluation of ML-VBLAST MIMO System using various antenna configurations with Ricean and Rayleigh Channel" Innovative Systems Design and Engineering, vol. 3, no. 10, pp. 62-71. 


\section{Biography}

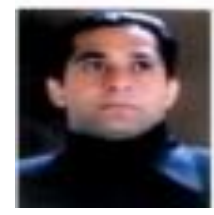

Mr. Amit Grover received his M.Tech degree in Electronics and Communication Engineering from Punjab Technical University, Kapurthla, Punjab, India in 2008 and received his B.Tech degree in Electronics and Communication Engineering from Punjab Technical University, Kapurthala, Punjab, India in 2001. His place of birth is Ferozepur, Punjab, India on $27^{\text {th }}$, September 1980. Currently, he is working as an Assistant Professor in Shaheed Bhagat Singh State Technical Campus, Ferozpur, Punjab, India. He has a working experience of 11 years in teaching. His area of interest includes signal processing, MIMO systems, Wireless mobile communication, High speed digital communications and 4G Wireless communications.

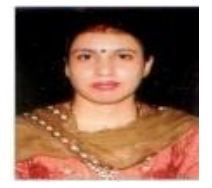

Ms. Neeti Grover received her master degree in Applied Sciences from Guru Nanak Dev University, Amritsar, and Punjab, India in 2007 and received her Bachelor's degree from Guru Nanak Dev University, Amritsar, Punjab, India in 2004. Her place of birth is Jallandhar, Punjab, India on $29^{\text {th }}$, December 1983. Currently, she is working as an Assistant Professor in the department of Applied Sciences and Humanities in Shaheed Bhagat Singh State Technical Campus (Poly Wing), Ferozpur, Punjab, India. 